Article

\title{
Design and Synthesis of $N$-phenyl Phthalimides as Potent Protoporphyrinogen Oxidase Inhibitors
}

\author{
Wei Gao, Xiaotian Li, Da Ren, Susu Sun, Jingqian Huo, Yanen Wang, Lai Chen * \\ and Jinlin Zhang *
}

Plant Protection College, Hebei Agricultural University, Baoding 071000, China; hbaugaow@163.com (W.G.); kddanongyao@163.com (X.L.); 18233470747@163.com (D.R.); susu8023d@163.com (S.S.); huojingqian@163.com (J.H.); yanenwang@163.com (Y.W.)

* Correspondence: chenlai@hebau.edu.cn (L.C.); zhangjinlin@hebau.edu.cn (J.Z.)

Received: 19 November 2019; Accepted: 27 November 2019; Published: 29 November 2019

\begin{abstract}
Protoporphyrinogen oxidase (PPO) has been identified as one of the most promising targets for herbicide discovery. A series of novel phthalimide derivatives were designed by molecular docking studies targeting the crystal structure of mitochondrial PPO from tobacco $(m t$ PPO, PDB: 1SEZ) by using Flumioxazin as a lead, after which the derivatives were synthesized and characterized, and their herbicidal activities were subsequently evaluated. The herbicidal bioassay results showed that compounds such as 3a (2-(4-bromo-2,6-difluorophenyl)isoindoline-1,3-dione), 3d (methyl 2-(4-chloro-1,3-dioxoisoindolin-2-yl)-5-fluorobenzoate), 3g (4-chloro-2-(5-methylisoxazol-3-yl) isoindoline-1,3-dione), 3j (4-chloro-2-(thiophen-2-ylmethyl)isoindoline-1,3-dione) and 3r (2-(4-bromo2,6-difluorophenyl)-4-fluoroisoindoline-1,3-dione) had good herbicidal activities; among them, 3a showed excellent herbicidal efficacy against $A$. retroflexus and B. campestris via the small cup method and via pre-emergence and post-emergence spray treatments. The efficacy was comparable to that of the commercial herbicides Flumioxazin, Atrazine, and Chlortoluron. Further, the enzyme activity assay results suggest that the mode of action of compound 3a involves the inhibition of the PPO enzyme, and 3a showed better inhibitory activity against PPO than did Flumioxazin. These results indicate that our molecular design strategy contributes to the development of novel promising PPO inhibitors.
\end{abstract}

Keywords: protoporphyrinogen oxidase; molecular design; N-phenyl phthalimides; herbicidal activity

\section{Introduction}

Protoporphyrinogen oxidase (PPO) is the last common enzyme in both chlorophyll (in plants) and haem (in animals) biosynthesis [1-4], catalyzing the oxidation of protoporphyrinogen IX to protoporphyrin IX via molecular oxygen [5-9], and this enzyme has been identified as one of the most significant targets for herbicide research $[10,11]$. During the last thirty years, a number of active compounds inhibiting the enzyme PPO have been synthesized [12,13], some of which have been developed for use as low-toxicity, efficient, broad-spectrum commercial herbicides [14,15], such as Flumioxazin [16,17], sulfentrazone [18] and saflufenacil [19].

Among the protox herbicides, $N$-phenyl phthalimides, which exhibit broad structural diversity [20], have attracted considerable attention; their representative commercial products, Cinidon-ethyl, Flumiclorac-pentyl and Flumioxazin, were identified as a result of a stepwise optimization procedure from Chlorphthalim. Their common structural feature consists of two parts: An N-substituted phenyl group and tetrahydrophthalimide, which can interact with key active centre residues of the 
$m t$ PPO enzyme, such as Arg98, Gly175, Leu372, Phe392, and FAD600, via H-bonding or $\pi-\pi$ stacking interactions [21]. These findings have contributed to the development of PPO inhibitors.

To obtain novel PPO inhibitors, a series of $N$-phenyl phthalimides were designed by molecular docking using the $m t \mathrm{PPO}$ as a target, and by using Flumioxazin as a lead (Figure 1) [22,23], after which they were synthesized and characterized by NMR and High resolution mass spectrometry (HR-MS). Their herbicidal activities were also evaluated against Brassica campestris (B. campestris), Amaranthus retroflexus (A. retroflexus) and Digitaria sanguinalis (D. sanguinalis) to verify our molecular design strategy.

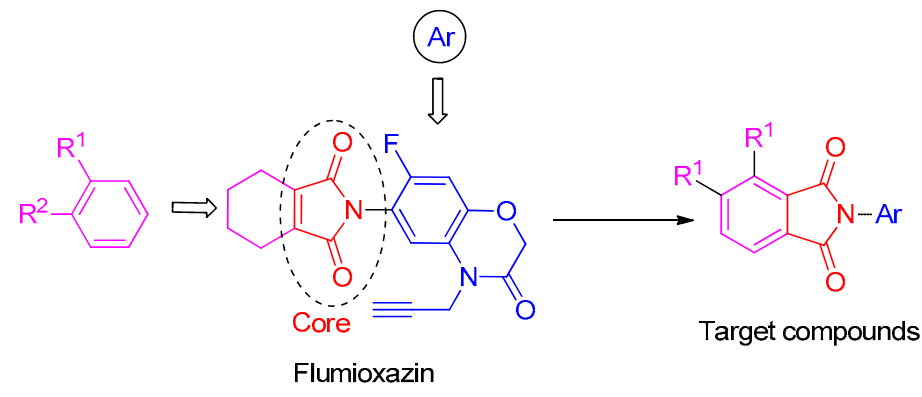

Figure 1. Design of the title compounds.

\section{Results and Discussion}

\subsection{Docking Analysis}

The affinity values of molecular docking between the ligand- $m+\mathrm{PPO}$ complexes were determined, which demonstrated that the affinity between $3 \mathbf{a}, 3 \mathbf{c}$ and $3 \mathbf{s}$ and $m t$ PPO exhibited the highest values with $-10.0,-10.1,10.2 \mathrm{Kcal} / \mathrm{mol}$ (Table 1), respectively, suggesting that these compounds may have good herbicidal activity. Their docking models were displayed by Pymol software (Figure 2), which indicated that no hydrogen bonding was found between these compounds and $m t \mathrm{PPO}$, which differs from the results of Flumioxazin (three hydrogen bonds). However, the phthalimide rings of compounds 3a, $3 \mathbf{c}$ and 3s shape $\pi-\pi$ stacking interactions with Phe392, which is conserved in plant PPO enzymes. The phenyl rings of compounds $\mathbf{3 a}, \mathbf{3} \mathbf{c}$ and $\mathbf{3}$ s were sandwiched by the residues Leu356 and Leu372. These results suggest that these compounds, with a phenyl ring substituting phthalimide rings, could be PPO inhibitors that exhibit good herbicidal activity.

Table 1. The affinity between compounds and $m t$ PPO. (Affinity, Kcal/mol).

\begin{tabular}{cccccccc}
\hline Compd. & Affinity & Compd. & Affinity & Compd. & Affinity & Compd. & Affinity \\
\hline 3a & -10.0 & $\mathbf{3 h}$ & -8.7 & $\mathbf{3 0}$ & -9.5 & $\mathbf{3 v}$ & -8.1 \\
$\mathbf{3 b}$ & -8.6 & $\mathbf{3 i}$ & -8.4 & $\mathbf{3} \mathbf{p}$ & -8.7 & $\mathbf{3 w}$ & -7.7 \\
$\mathbf{3} \mathbf{c}$ & -10.1 & $\mathbf{3 j}$ & -7.7 & $\mathbf{3 q}$ & -9.3 & $\mathbf{3 x}$ & -9.8 \\
$\mathbf{3 d}$ & -9.1 & $\mathbf{3 k}$ & -9.4 & $\mathbf{3 r}$ & -9.0 & $\mathbf{3 y}$ & -9.5 \\
$\mathbf{3 e}$ & -9.8 & $\mathbf{3 1}$ & -8.9 & $\mathbf{3 s}$ & -10.2 & Flumioxazin & -9.5 \\
$\mathbf{3 f}$ & -9.7 & $\mathbf{3} \mathbf{m}$ & -9.9 & $\mathbf{3 t}$ & -9.7 & Chlortoluron & -7.1 \\
\hline
\end{tabular}



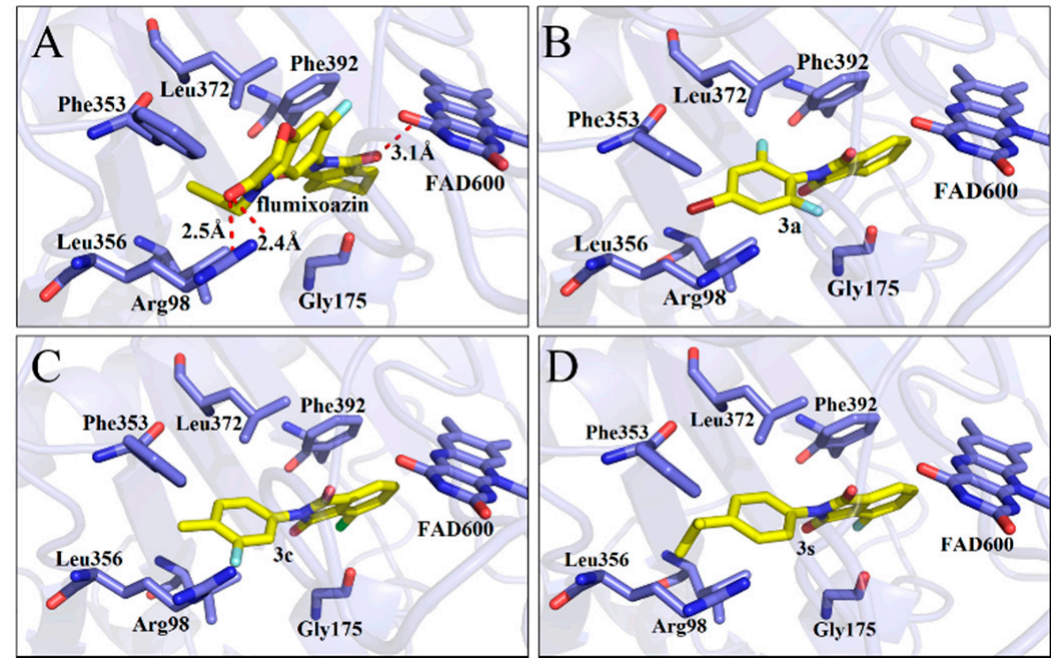

Figure 2. Docking model shown in Pymol, molecule (blade yellowing) and residues of $m t \mathrm{PPO}$ (wrinkling); Flumioxazin (A), 3a (B), 3c (C), and 3s (D).

\subsection{Chemistry}

The starting material 1 and 2 could be commercially available. Compounds 3 were prepared by nucleophilic substitution reaction between phthalic anhydrides $\mathbf{1}$ and amines $\mathbf{2}$ in glacial acetic acid, with yields ranging from $28 \%$ to $87 \%$ (Table 2). The reaction solvent and temperature were the key conditions for the reaction yield. The yield of the reaction stirred at $110{ }^{\circ} \mathrm{C}$ was much better than that of $80^{\circ} \mathrm{C}$. Besides, the yield of the reaction in glacial acetic acid was higher than that of other solvents, such as ethanol. 
Table 2. General Synthetic Route for Compounds 3a-3y.

\begin{tabular}{|c|c|c|c|c|c|c|c|c|c|c|c|c|c|c|}
\hline & & & & & 1 & & 2 & & 3 & & & & & \\
\hline Compd. & $\mathbf{R}^{1}$ & $\mathbf{R}^{2}$ & Ar & Yield (\%) & Compd. & $\mathbf{R}^{1}$ & $\mathbf{R}^{2}$ & Ar & Yield (\%) & Compd. & $\mathbf{R}^{1}$ & $\mathbf{R}^{2}$ & $\mathrm{Ar}$ & Yield (\%) \\
\hline $3 a$ & $\mathrm{H}$ & $\mathrm{H}$ & & 73 & $3 \mathbf{j}$ & $\mathrm{Cl}$ & $\mathrm{H}$ & & 71 & $3 \mathrm{~s}$ & $\mathrm{~F}$ & $\mathrm{H}$ & & 79 \\
\hline $3 b$ & $\mathrm{H}$ & $\mathrm{Cl}$ & & 77 & $3 k$ & $\mathrm{Cl}$ & $\mathrm{H}$ & & 63 & $3 t$ & $\mathrm{H}$ & $\mathrm{Cl}$ & & 79 \\
\hline $3 c$ & $\mathrm{Cl}$ & $\mathrm{H}$ & & 81 & 31 & $\mathrm{Cl}$ & $\mathrm{H}$ & & 86 & $3 \mathbf{u}$ & $\mathrm{H}$ & $\mathrm{Cl}$ & & 70 \\
\hline $3 d$ & $\mathrm{Cl}$ & $\mathrm{H}$ & & 28 & $3 \mathrm{~m}$ & $\mathrm{Cl}$ & $\mathrm{H}$ & & 84 & $3 v$ & $\mathrm{H}$ & $\mathrm{Cl}$ & & 76 \\
\hline $3 e$ & $\mathrm{Cl}$ & $\mathrm{H}$ & & 80 & $3 n$ & $\mathrm{Cl}$ & $\mathrm{H}$ & & 52 & $3 w$ & $\mathrm{H}$ & $\mathrm{Cl}$ & & 79 \\
\hline $3 \mathrm{f}$ & $\mathrm{Cl}$ & $\mathrm{H}$ & & 78 & 30 & $\mathrm{Cl}$ & $\mathrm{H}$ & & 87 & $3 x$ & $\mathrm{H}$ & $\mathrm{CH}_{3}$ & & 47 \\
\hline $3 g$ & $\mathrm{Cl}$ & $\mathrm{H}$ & & 58 & $3 p$ & $\mathrm{Cl}$ & $\mathrm{H}$ & & 30 & $3 y$ & $\mathrm{H}$ & $\mathrm{NO}_{2}$ & & 57 \\
\hline $3 \mathrm{~h}$ & $\mathrm{Cl}$ & $\mathrm{H}$ & & 60 & $3 q$ & $\mathrm{~F}$ & $\mathrm{H}$ & & 57 & & & & & \\
\hline $3 \mathbf{i}$ & $\mathrm{Cl}$ & $\mathrm{H}$ & & 67 & $3 r$ & $\mathrm{~F}$ & $\mathrm{H}$ & & 78 & & & & & \\
\hline
\end{tabular}




\subsection{Herbicidal Activity}

The herbicidal activity against B. campestris, A. retroflexus and D. sanguinalis at $200 \mathrm{mg} / \mathrm{L}$ of compound 3 were evaluated by the small cup method, and the results are shown in Table 3. The data showed that 3a at $200 \mathrm{mg} / \mathrm{L}$ displayed 92\% growth inhibition against B. campestris roots, which was better than that of the positive controls Chlortoluron (85\%), Atrazine (80\%), and Flumioxazin (85\%); 3a also showed $61 \%$ growth inhibition against stems of B. campestris, which was better than that of Atrazine (51\%) and similar to that of Chlortoluron (58\%). During the test, the B. campestris plants treated with 3a became chlorotic, and blade yellowing was also observed. In addition, with $87 \%$ growth inhibition, 3a exhibited better efficacy against $A$. retroflexus stems than did Atrazine, which was similar to that of Flumioxazin (88\%); the leaves of $A$. retroflexus were wrinkled. Furthermore, 3a displayed $68 \%$ and $83 \%$ growth inhibition against the roots and stems of $D$. sanguinalis, respectively, which were better than the inhibition due to Atrazine. Compounds $\mathbf{3 d}$ and $\mathbf{3 o}$ exhibited high activity against the roots of $A$. retroflexus, with $81 \%$ and $83 \%$ growth inhibition, respectively, while Atrazine showed only $32 \%$ inhibition under the same conditions; 30 also showed $89 \%$ growth inhibition activity against $B$. campestris roots, which was slightly better than that of the three positive controls. $\mathbf{3 g}$ showed excellent efficacy against D. sanguinalis roots and stems, with $91 \%$ and $83 \%$ growth inhibition, respectively, these percentages were better than those due to Atrazine.

Table 3. Herbicidal activity of compounds $\mathbf{3 a}-3 \mathbf{y}$ at $200 \mathrm{mg} / \mathrm{L}$ by the small cup method.

\begin{tabular}{|c|c|c|c|c|c|c|}
\hline \multirow{2}{*}{ Compd. } & \multicolumn{2}{|c|}{$B C^{a}$} & \multicolumn{2}{|c|}{$A R^{\mathrm{a}}$} & \multicolumn{2}{|c|}{$D S^{\mathrm{a}}$} \\
\hline & Root & Stem & Root & Stem & Root & Stem \\
\hline $3 a$ & $92 \pm 1$ & $61 \pm 2$ & $37 \pm 1$ & $87 \pm 2$ & $68 \pm 3$ & $83 \pm 2$ \\
\hline $3 b$ & $74 \pm 2$ & 0 & $28 \pm 4$ & $66 \pm 4$ & $77 \pm 2$ & $62 \pm 3$ \\
\hline $3 c$ & $78 \pm 5$ & 0 & $65 \pm 1$ & $22 \pm 1$ & $61 \pm 1$ & 0 \\
\hline $3 d$ & $82 \pm 1$ & $35 \pm 1$ & $81 \pm 4$ & $33 \pm 2$ & $65 \pm 2$ & $10 \pm 1$ \\
\hline $3 e$ & $28 \pm 2$ & $21 \pm 2$ & $51 \pm 1$ & $55 \pm 3$ & $28 \pm 2$ & $38 \pm 3$ \\
\hline $3 f$ & $46 \pm 2$ & $73 \pm 3$ & $68 \pm 0$ & $42 \pm 0$ & $47 \pm 3$ & 0 \\
\hline $3 g$ & 0 & $18 \pm 0$ & $74 \pm 2$ & $41 \pm 2$ & $91 \pm 1$ & $83 \pm 1$ \\
\hline $3 h$ & $77 \pm 3$ & 0 & $62 \pm 2$ & $33 \pm 1$ & $55 \pm 1$ & $34 \pm 1$ \\
\hline $3 \mathbf{i}$ & $58 \pm 4$ & 0 & $55 \pm 1$ & $27 \pm 2$ & $67 \pm 5$ & $17 \pm 3$ \\
\hline $3 \mathbf{j}$ & $75 \pm 0$ & 0 & $66 \pm 1$ & $34 \pm 1$ & $87 \pm 2$ & $11 \pm 0$ \\
\hline $3 k$ & $65 \pm 1$ & 0 & $53 \pm 3$ & $36 \pm 2$ & $57 \pm 1$ & 0 \\
\hline 31 & $68 \pm 2$ & $6 \pm 2$ & $44 \pm 3$ & $31 \pm 2$ & $48 \pm 4$ & $51 \pm 3$ \\
\hline $3 m$ & $45 \pm 3$ & 0 & $55 \pm 4$ & $45 \pm 3$ & $57 \pm 2$ & 0 \\
\hline $3 n$ & $49 \pm 1$ & 0 & $59 \pm 2$ & $33 \pm 2$ & $59 \pm 4$ & 0 \\
\hline 30 & $89 \pm 1$ & $77 \pm 0$ & $83 \pm 5$ & $51 \pm 3$ & $63 \pm 4$ & 0 \\
\hline $3 p$ & $51 \pm 2$ & $9 \pm 2$ & $37 \pm 3$ & $72 \pm 4$ & $58 \pm 1$ & $45 \pm 2$ \\
\hline $3 q$ & $28 \pm 1$ & $22 \pm 1$ & $22 \pm 1$ & $21 \pm 2$ & $38 \pm 3$ & 0 \\
\hline $3 r$ & $65 \pm 4$ & $54 \pm 3$ & $62 \pm 2$ & $75 \pm 3$ & $87 \pm 2$ & $86 \pm 2$ \\
\hline $3 s$ & $64 \pm 2$ & $15 \pm 1$ & $35 \pm 4$ & $61 \pm 5$ & $53 \pm 3$ & $28 \pm 5$ \\
\hline $3 t$ & $54 \pm 1$ & 0 & $16 \pm 1$ & $39 \pm 4$ & $46 \pm 2$ & $18 \pm 1$ \\
\hline $3 \mathbf{u}$ & $77 \pm 1$ & $53 \pm 3$ & 0 & $53 \pm 2$ & $51 \pm 2$ & $32 \pm 1$ \\
\hline $3 \mathbf{v}$ & $60 \pm 5$ & 0 & $10 \pm 2$ & $42 \pm 3$ & $22 \pm 1$ & $21 \pm 2$ \\
\hline $3 w$ & $58 \pm 2$ & 0 & $28 \pm 1$ & $61 \pm 2$ & $79 \pm 2$ & $11 \pm 2$ \\
\hline $3 x$ & $59 \pm 4$ & 0 & $36 \pm 3$ & $55 \pm 3$ & $31 \pm 2$ & $24 \pm 1$ \\
\hline $3 y$ & $68 \pm 2$ & $9 \pm 0$ & $34 \pm 4$ & $60 \pm 4$ & $44 \pm 1$ & $37 \pm 3$ \\
\hline Chlortoluron & $85 \pm 4$ & $58 \pm 3$ & $92 \pm 3$ & $90 \pm 5$ & $98 \pm 0$ & $97 \pm 1$ \\
\hline Atrazine & $81 \pm 1$ & $52 \pm 1$ & $32 \pm 2$ & $66 \pm 2$ & $58 \pm 1$ & $60 \pm 2$ \\
\hline Flumioxazin & $85 \pm 3$ & $72 \pm 5$ & $82 \pm 2$ & $88 \pm 1$ & $71 \pm 2$ & $91 \pm 0$ \\
\hline
\end{tabular}

${ }^{\text {a }} B C$ for B. campestris; $A R$ for $A$. retroflexus; $D S$ for D. sanguinalis.

The results of the evaluation of the post-emergence herbicidal activities are shown in Table 4 . The data showed that compounds $3 \mathrm{a}$ and $\mathbf{3 d}$ exhibited $82 \%$ and $73 \%$ fresh weight growth inhibition at $90 \mathrm{~g}$ ai/ha against $A$. retroflexus, respectively. Therefore, 3a and $\mathbf{3 d}$ were also chosen for further 
pre-emergence herbicidal activity tests. As shown in Table 5, 3a at $90 \mathrm{~g}$ ai/ha exhibited a $98 \%$ inhibitory effect against $A$. retroflexus, and the effects were not significantly different from those of Flumioxazin.

Table 4. Post-emergence herbicidal activity of compounds $3 a-3 y$ at $90 \mathrm{~g}$ ai/ha.

\begin{tabular}{|c|c|c|c|c|c|c|c|}
\hline Compd. & $B C$ & $A R$ & $D S$ & Compd. & $B C$ & $A R$ & $D S$ \\
\hline $3 a$ & $25 \pm 2$ & $82 \pm 3$ & $37 \pm 1$ & 30 & $16 \pm 2$ & $27 \pm 2$ & $23 \pm 2$ \\
\hline $3 b$ & $27 \pm 2$ & $18 \pm 2$ & $12 \pm 1$ & $3 p$ & $19 \pm 4$ & $20 \pm 1$ & $14 \pm 0$ \\
\hline $3 c$ & $48 \pm 1$ & $13 \pm 1$ & $19 \pm 3$ & $3 q$ & $30 \pm 2$ & $19 \pm 2$ & $36 \pm 1$ \\
\hline $3 d$ & $60 \pm 3$ & $73 \pm 2$ & $20 \pm 1$ & $3 r$ & $37 \pm 1$ & $22 \pm 3$ & $36 \pm 5$ \\
\hline $3 e$ & $35 \pm 4$ & $47 \pm 3$ & $33 \pm 1$ & $3 s$ & $22 \pm 1$ & $44 \pm 2$ & $18 \pm 1$ \\
\hline $3 f$ & $56 \pm 1$ & $10 \pm 2$ & $11 \pm 1$ & $3 t$ & $29 \pm 2$ & $62 \pm 3$ & $17 \pm 1$ \\
\hline $3 g$ & $38 \pm 2$ & 0 & $23 \pm 2$ & $3 \mathbf{u}$ & $60 \pm 2$ & $5 \pm 1$ & $28 \pm 2$ \\
\hline $3 h$ & $58 \pm 1$ & $7 \pm 1$ & $27 \pm 2$ & $3 v$ & $33 \pm 1$ & 0 & 0 \\
\hline $3 \mathbf{i}$ & $28 \pm 2$ & $34 \pm 2$ & $37 \pm 1$ & $3 w$ & $35 \pm 1$ & $22 \pm 4$ & $36 \pm 4$ \\
\hline $3 \mathbf{j}$ & $23 \pm 2$ & $20 \pm 5$ & $31 \pm 1$ & $3 x$ & $17 \pm 2$ & $7 \pm 2$ & $7 \pm 1$ \\
\hline $3 k$ & $35 \pm 5$ & $24 \pm 3$ & 0 & $3 y$ & $28 \pm 1$ & 0 & 0 \\
\hline 31 & $33 \pm 1$ & $37 \pm 2$ & $26 \pm 2$ & Chlortoluron & $\mathrm{nd}^{\mathrm{a}}$ & $91 \pm 1$ & $65 \pm 2$ \\
\hline $3 m$ & $22 \pm 4$ & $35 \pm 1$ & $34 \pm 2$ & Atrazine & $\mathrm{nd}^{\mathrm{a}}$ & $91 \pm 2$ & $47 \pm 3$ \\
\hline $3 n$ & $25 \pm 2$ & $30 \pm 5$ & $11 \pm 1$ & Flumioxazin & $85 \pm 6$ & $92 \pm 4$ & $86 \pm 6$ \\
\hline
\end{tabular}

Table 5. Per-emergence herbicidal activity of compounds 3a, 3d and Flumioxazin at $90 \mathrm{~g}$ ai/ha.

\begin{tabular}{ccc}
\hline Compd. & $A R$ & DS \\
\hline 3a & $98 \pm 2$ & $61 \pm 2$ \\
3d & $36 \pm 3$ & 0 \\
Flumioxazin & 100 & 100 \\
\hline
\end{tabular}

In summary, 3a exhibited excellent herbicidal activity and should be further developed. Moreover, its herbicidal activity conformed to the predictions of the molecular docking studies.

\subsection{Crystal Structure Determination of Compound $3 a$}

The X-ray diffraction structure of compound 3a, cultured from the mixture of ethanol and chloroform, was shown in Figure 3, and the data were available at the Cambridge Crystallographic Data Centre (CCDC 1923372).

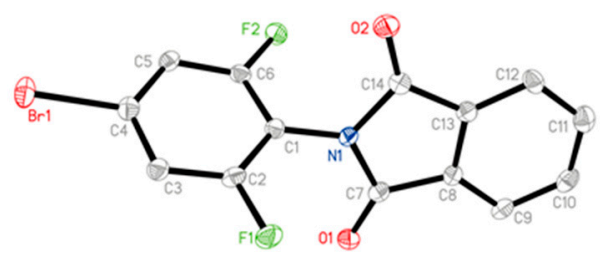

Figure 3. Crystal structure for 3a by X-ray diffraction determination.

\subsection{PPO Enzyme Assays}

The PPO enzyme activity of $D$. sanguinalis, treated with 3 a and Flumioxazin at $90 \mathrm{~g}$ ai/ha, were measured, and the control was sprayed with blank solution without any compounds. The results, shown in Figure 4, indicated that the PPO activity values of the 3a treatment and the control Flumioxazin were affected; in addition, 3a showed a stronger effect on the PPO enzyme, with 33.01\%, compared to that of the positive control Flumioxazin, with $21.80 \%$. 


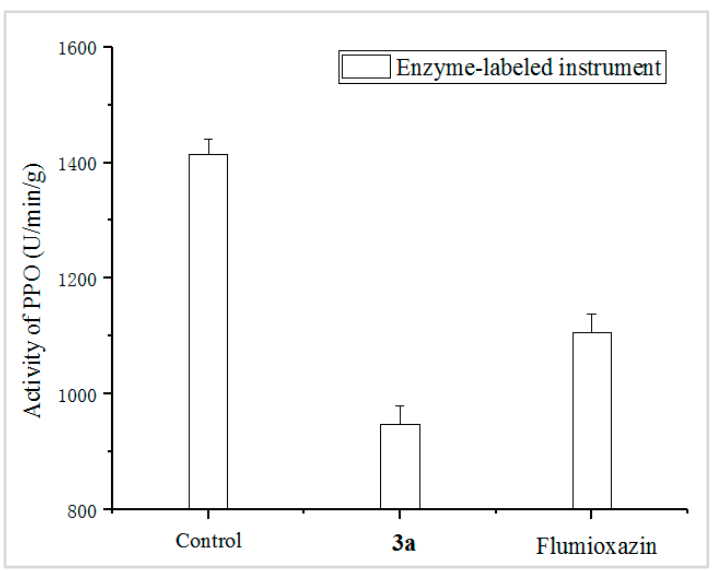

Figure 4. PPO enzyme activity involved by 3a and Flumioxazin. (The control was sprayed with blank solution without any compounds; the $\mathbf{3}$ a and Flumioxazin were sprayed with $3 \mathbf{a}$ and Flumioxazin at $90 \mathrm{~g}$ ai/ha, respectively).

\section{Materials and Methods}

\subsection{Molecular Docking}

The structure of $m t \mathrm{PPO}$ was available at the National Center for Biotechnology Information (NCBI) database. The modelled complexes of $m t \mathrm{PPO}$ and ligands (Flumioxazin, Chlorotoluron, Atrazine and designed target compounds) were prepared by using AutoDockTools 1.5.6 (Molecular Graphics Laboratory, La Jolla, CA 92037-1000, USA) and analysed via the AutoDock Vina program (Molecular Graphics Laboratory, La Jolla, CA 92037-1000, USA) [24,25]. The structures of the complexes were generated by the Pymol tool 2.2.0 [26,27].

\subsection{Equipment and Materials}

The melting points of the new compounds were measured in a microfusion melting point apparatus (X-4) (Taike, Beijing, China) and uncorrected. ${ }^{1} \mathrm{H}-\mathrm{NMR}$ and ${ }^{13} \mathrm{C}-\mathrm{NMR}$ spectra were recorded on Varian 400 spectrometer at $400 \mathrm{MHz}$ and $101 \mathrm{MHz}$ using tetramethylsilane as internal standard (solvent $\mathrm{CDCl}_{3}$ or DMSO- $d_{6}$ ). HR-MS date were detected on an FTICR-MS Varian 7.0T FTICR-MS equipment (Agilent, Lexington, MA, USA). Crystal structure was recorded on a Bruker SMART 1000CCD diffraction meter.

\subsection{General Synthetic Procedure for Compounds 3}

The starting materials $\mathbf{1}$ and $\mathbf{2}$ were commercially available (Energy Chemical, Shanghai, China). Compound $\mathbf{2}(3.72 \mathrm{mmol})$ was added to a stirred solution of compound $\mathbf{1}(3.38 \mathrm{mmol})$ in glacial acetic acid $(10 \mathrm{~mL})$. The reaction mixture was then stirred at $110^{\circ} \mathrm{C}$ for $4 \mathrm{~h}$. After completion of the reaction, the solvent was evaporated, and the residue was purified on a silica gel column chromatography and eluted with ethyl acetate/petroleum ether $\left(\mathrm{bp} 60-90^{\circ} \mathrm{C}\right)(1: 3, v / v)$ to give compounds 3 . Among them, compounds $\mathbf{3 k}, \mathbf{3 1}, \mathbf{3 n}, \mathbf{3} \mathbf{x}$ and $\mathbf{3} \mathbf{y}$ were published [28-32], while their herbicidal activities were not studied. Their yields, physical properties, ${ }^{1} \mathrm{H}-\mathrm{NMR},{ }^{13} \mathrm{C}-\mathrm{NMR}$, and HR-MS results are shown as follows:

Data for 3a (2-(4-bromo-2,6-difluorophenyl)isoindoline-1,3-dione): white solid; yield, 73\%; m.p.: $175-176{ }^{\circ} \mathrm{C} ;{ }^{1} \mathrm{H}-\mathrm{NMR}\left(400 \mathrm{MHz}, \mathrm{CDCl}_{3}\right) \delta 7.98(\mathrm{dd}, J=5.5,3.1 \mathrm{~Hz}, 2 \mathrm{H}), 7.83(\mathrm{dd}, J=5.5,3.1 \mathrm{~Hz}$, 2H), $7.29(\mathrm{~d}, J=6.7 \mathrm{~Hz}, 2 \mathrm{H}) \cdot{ }^{13} \mathrm{C}-\mathrm{NMR}\left(101 \mathrm{MHz}, \mathrm{CDCl}_{3}\right) \delta 165.41(\mathrm{~s}), 160.09(\mathrm{~s}), 157.47(\mathrm{~s}), 134.73(\mathrm{~s})$, 131.93 (s), 124.22 (s), 123.29 (s), 116.52 (s), 116.25 (s). HR-MS (ESI) [M + H] calcd for $\mathrm{C}_{14} \mathrm{H}_{6} \mathrm{BrF}_{2} \mathrm{NO}_{2}$ : 337.9500, found: 337.9621 .

Data for $\mathbf{3 b}$ (5-chloro-2-(5-methylisoxazol-3-yl)isoindoline-1,3-dione): white solid; yield, 77\%; m.p.: $164-165^{\circ} \mathrm{C} ;{ }^{1} \mathrm{H}-\mathrm{NMR}\left(400 \mathrm{MHz}, \mathrm{CDCl}_{3}\right) \delta 7.95(\mathrm{dd}, J=12.4,4.8 \mathrm{~Hz}, 2 \mathrm{H}), 7.79(\mathrm{dd}, J=8.0,1.7 \mathrm{~Hz}$, 1H), $6.48(\mathrm{~s}, 1 \mathrm{H}), 2.51(\mathrm{~s}, 3 \mathrm{H}) .{ }^{13} \mathrm{C}-\mathrm{NMR}\left(101 \mathrm{MHz}, \mathrm{CDCl}_{3}\right) \delta 170.98(\mathrm{~s}), 163.90$ (s), 163.65 (s), 153.28 
(s), 141.86 (s), 135.13 (s), 133.13 (s), 129.55 (s), 125.56 (s), 124.67 (s), 97.96 (s), 12.73 (s). HR-MS (ESI) $[\mathrm{M}+\mathrm{H}]^{+}$calcd for $\mathrm{C}_{12} \mathrm{H}_{7} \mathrm{~N}_{2} \mathrm{O}_{3}: 263.0145$, found: 263.0223 .

Data for 3c (4-chloro-2-(3-fluoro-4-methylphenyl)isoindoline-1,3-dione): white solid; yield, 81\%; m.p.: $197-198^{\circ} \mathrm{C}^{1}{ }^{1} \mathrm{H}-\mathrm{NMR}\left(400 \mathrm{MHz}, \mathrm{CDCl}_{3}\right) \delta 7.95-7.80(\mathrm{~m}, 1 \mathrm{H}), 7.79-7.60(\mathrm{~m}, 2 \mathrm{H}), 7.30(\mathrm{~d}, J=8.1 \mathrm{~Hz}$, $1 \mathrm{H}), 7.14(\mathrm{t}, J=8.3 \mathrm{~Hz}, 2 \mathrm{H}), 2.32(\mathrm{~d}, J=7.1 \mathrm{~Hz}, 3 \mathrm{H}) .{ }^{13} \mathrm{C}-\mathrm{NMR}\left(101 \mathrm{MHz}, \mathrm{CDCl}_{3}\right) \delta 165.46(\mathrm{~s}), 162.00(\mathrm{~s})$, 159.55 (s), 136.11 (s), 135.23 (s), 133.58 (s), 131.88 (s), 131.49 (s), 129.90 (s), 127.14 (s), 125.23 (s), 122.18 (s), $121.74(\mathrm{~s}), 113.59$ (s), 14.27 (s). HR-MS (ESI) [M + H] ${ }^{+}$calcd for $\mathrm{C}_{15} \mathrm{H}_{9} \mathrm{ClFNO}_{2}: 290.0306$, found: 290.0380 .

Data for 3d (methyl 2-(4-chloro-1,3-dioxoisoindolin-2-yl)-5-fluorobenzoate): white solid; yield, 28\%; m.p.: $136-137^{\circ} \mathrm{C} ;{ }^{1} \mathrm{H}-\mathrm{NMR}\left(400 \mathrm{MHz}, \mathrm{CDCl}_{3}\right) \delta 7.89(\mathrm{~d}, J=3.8 \mathrm{~Hz}, 2 \mathrm{H}), 7.74(\mathrm{~d}, J=3.8 \mathrm{~Hz}, 2 \mathrm{H}), 7.40$ $(\mathrm{d}, J=4.6 \mathrm{~Hz}, 2 \mathrm{H}), 3.80(\mathrm{~s}, 3 \mathrm{H}) .{ }^{13} \mathrm{C}-\mathrm{NMR}\left(101 \mathrm{MHz}, \mathrm{CDCl}_{3}\right) \delta 165.96(\mathrm{~s}), 164.93(\mathrm{~s}), 163.85$ (s), $160.96(\mathrm{~s})$, 136.04 (s), 135.19 (s), 134.03 (s), 132.32 (s), 131.86 (s), 129.68 (s), 127.72 (s), 127.45 (s), 122.25 (s), 120.48 (s), 118.88 (s), 52.62 (s). HR-MS (ESI) [M + H] ${ }^{+}$calcd for $\mathrm{C}_{16} \mathrm{H}_{9} \mathrm{ClFNO}_{4}$ : 334.0204 , found: 334.0273.

Data for 3e (2-(4-bromo-2,6-difluorophenyl)-4-chloroisoindoline-1,3-dione): white solid; yield, 80\%; m.p.: $185-186{ }^{\circ} \mathrm{C} ;{ }^{1} \mathrm{H}-\mathrm{NMR}\left(400 \mathrm{MHz}, \mathrm{CDCl}_{3}\right) \delta 7.94-7.82(\mathrm{~m}, 2 \mathrm{H}), 7.74(\mathrm{~d}, J=3.8 \mathrm{~Hz}, 2 \mathrm{H})$, 7.45-7.35 (m, 2H), $3.80(\mathrm{~s}, 3 \mathrm{H}) .{ }^{13} \mathrm{C}-\mathrm{NMR}\left(101 \mathrm{MHz}, \mathrm{CDCl}_{3}\right) \delta 163.97$ (s), 162.87 (s), 159.98 (s), 157.41 (s), $136.47(\mathrm{~s}), 135.60$ (s), 133.91 (s), 132.39 (s), 127.72 (s), 123.57 (s), 122.67 (s), 116.55 (s), 116.28 (s), $108.22(\mathrm{~s})$. HR-MS (ESI) $[\mathrm{M}+\mathrm{H}]^{+}$calcd for $\mathrm{C}_{14} \mathrm{H}_{5} \mathrm{BrClF}_{2} \mathrm{NO}_{2}$ : 371.9160, found: 371.9232 .

Data for $3 f$ (4-chloro-2-(4-isopropylphenyl)isoindoline-1,3-dione): white solid; yield, 78\%; m.p.: 154-155 ${ }^{\circ} \mathrm{C} ;{ }^{1} \mathrm{H}-\mathrm{NMR}\left(400 \mathrm{MHz}, \mathrm{CDCl}_{3}\right) \delta 7.89(\mathrm{dd}, J=4.4,3.9 \mathrm{~Hz}, 1 \mathrm{H}), 7.76-7.68(\mathrm{~m}, 2 \mathrm{H}), 7.43-7.32(\mathrm{~m}$, $4 \mathrm{H}), 2.99(\mathrm{~s}, 1 \mathrm{H}), 1.31(\mathrm{~d}, J=6.9 \mathrm{~Hz}, 6 \mathrm{H}) .{ }^{13} \mathrm{C}-\mathrm{NMR}\left(101 \mathrm{MHz}, \mathrm{CDCl}_{3}\right) \delta 166.04(\mathrm{~s}), 165.03(\mathrm{~s}), 149.13(\mathrm{~s})$, 136.09 (s), 135.22 (s), 133.93 (s), 131.85 (s), 128.83 (s), 127.46 (s), 127.25 (s), 126.42 (s), 122.21 (s), 33.95 (s), 23.93 (s). HR-MS (ESI) [M+H] ${ }^{+}$calcd for $\mathrm{C}_{17} \mathrm{H}_{14} \mathrm{ClNO}_{2}$ : 300.0713, found: 300.0791 .

Data for 3g (4-chloro-2-(5-methylisoxazol-3-yl)isoindoline-1,3-dione): White solid; yield, 58\%; m.p.: $160-161{ }^{\circ} \mathrm{C} ;{ }^{1} \mathrm{H}-\mathrm{NMR}\left(400 \mathrm{MHz}, \mathrm{CDCl}_{3}\right) \delta 7.95-7.89(\mathrm{~m}, 1 \mathrm{H}), 7.76(\mathrm{~d}, J=4.4 \mathrm{~Hz}, 2 \mathrm{H}), 6.49$ (s, 1H), $2.52(\mathrm{~s}, 3 \mathrm{H}) .{ }^{13} \mathrm{C}-\mathrm{NMR}\left(101 \mathrm{MHz}, \mathrm{CDCl}_{3}\right) \delta 170.96(\mathrm{~s}), 163.34(\mathrm{~s}), 162.52(\mathrm{~s}), 153.18(\mathrm{~s}), 136.69$ (s), $135.83(\mathrm{~s}), 133.59$ (s), 132.52 (s), 127.21 (s), 122.81 (s), 98.09 (s), 12.76 (s). HR-MS (ESI) [M + H] ${ }^{+}$calcd for $\mathrm{C}_{12} \mathrm{H}_{7} \mathrm{ClN}_{2} \mathrm{O}_{3}$ : 263.0145, found: 263.0220 .

Data for 3h (4-chloro-2-(1-methyl-1H-pyrazol-5-yl)isoindoline-1,3-dione): white solid; yield, 60\%; m.p.: $195-196{ }^{\circ} \mathrm{C} ;{ }^{1} \mathrm{H}-\mathrm{NMR}\left(400 \mathrm{MHz}, \mathrm{CDCl}_{3}\right) \delta 7.91(\mathrm{dd}, J=4.6,3.7 \mathrm{~Hz}, 1 \mathrm{H}), 7.81-7.74(\mathrm{~m}, 2 \mathrm{H}), 7.60(\mathrm{~d}$, $J=2.0 \mathrm{~Hz}, 1 \mathrm{H}), 6.33(\mathrm{~d}, J=2.0 \mathrm{~Hz}, 1 \mathrm{H}), 3.78(\mathrm{~s}, 3 \mathrm{H}) .{ }^{13} \mathrm{C}-\mathrm{NMR}\left(101 \mathrm{MHz}, \mathrm{CDCl}_{3}\right) \delta 164.65(\mathrm{~s}), 163.59(\mathrm{~s})$, 138.84 (s), 136.69 (s), 135.83 (s), 133.61 (s), 132.52 (s), 129.13 (s), 127.39 (s), 122.76 (s), 104.67 (s), 36.51 (s). HR-MS (ESI) $[\mathrm{M}+\mathrm{H}]^{+}$calcd for $\mathrm{C}_{12} \mathrm{H}_{8} \mathrm{ClN}_{3} \mathrm{O}_{2}: 262.0305$, found: 262.0379 .

Data for 3i (4-chloro-2-(3,4-dichlorobenzyl)isoindoline-1,3-dione): white solid; yield, 67\%; m.p.: $143-144{ }^{\circ} \mathrm{C} ;{ }^{1} \mathrm{H}-\mathrm{NMR}\left(400 \mathrm{MHz}, \mathrm{CDCl}_{3}\right) \delta 7.78(\mathrm{dd}, J=4.8,3.4 \mathrm{~Hz}, 1 \mathrm{H}), 7.69-7.62(\mathrm{~m}, 2 \mathrm{H}), 7.53(\mathrm{~d}$, $J=2.0 \mathrm{~Hz}, 1 \mathrm{H}), 7.39(\mathrm{~d}, J=8.2 \mathrm{~Hz}, 1 \mathrm{H}), 7.30(\mathrm{~d}, J=2.0 \mathrm{~Hz}, 1 \mathrm{H}), 4.78(\mathrm{~s}, 2 \mathrm{H}) .{ }^{13} \mathrm{C}-\mathrm{NMR}(101 \mathrm{MHz}$, $\left.\mathrm{CDCl}_{3}\right) \delta 166.32(\mathrm{~s}), 165.47(\mathrm{~s}), 135.99(\mathrm{~d}, J=4.7 \mathrm{~Hz}), 135.18(\mathrm{~s}), 133.97(\mathrm{~s}), 132.77(\mathrm{~s}), 132.30(\mathrm{~s}), 131.65(\mathrm{~s})$, $130.76(\mathrm{~d}, J=8.8 \mathrm{~Hz}), 128.30(\mathrm{~s}), 127.63(\mathrm{~s}), 122.05(\mathrm{~s}), 40.70(\mathrm{~s})$. HR-MS (ESI) [M + H] ${ }^{+}$calcd for $\mathrm{C}_{15} \mathrm{H}_{8} \mathrm{Cl}_{3} \mathrm{NO}_{2}$ : 339.9621, found: 339.9692 .

Data for 3j (4-chloro-2-(thiophen-2-ylmethyl)isoindoline-1,3-dione): white solid; yield, 71\%; m.p.: $135-136{ }^{\circ} \mathrm{C} ;{ }^{1} \mathrm{H}-\mathrm{NMR}\left(400 \mathrm{MHz}, \mathrm{CDCl}_{3}\right) \delta 7.78(\mathrm{dd}, J=4.6,3.6 \mathrm{~Hz}, 1 \mathrm{H}), 7.68-7.61(\mathrm{~m}, 2 \mathrm{H}), 7.31-7.15$ $(\mathrm{m}, 2 \mathrm{H}), 6.95(\mathrm{dd}, J=5.1,3.6 \mathrm{~Hz}, 1 \mathrm{H}), 5.03(\mathrm{~s}, 2 \mathrm{H}) .{ }^{13} \mathrm{C}-\mathrm{NMR}\left(101 \mathrm{MHz}, \mathrm{CDCl}_{3}\right) \delta 166.09(\mathrm{~s}), 165.20(\mathrm{~s})$, $137.56(\mathrm{~s}), 135.81$ (s), $135.03(\mathrm{~s}), 134.09$ (s), 131.54 (s), 128.08 (s), 127.74 (s), 126.95 (s), 126.08 (s), 121.95 (s), 35.82 (s). HR-MS (ESI) [M + H] ${ }^{+}$calcd for $\mathrm{C}_{13} \mathrm{H}_{8} \mathrm{ClNO}_{2} \mathrm{~S}: 277.9964$, found: 278.0038 .

Data for 3k (4-chloro-2-(3-fluoro-4-nitrophenyl)isoindoline-1,3-dione): brown solid, yield, 63\%; m.p.: $231-232{ }^{\circ} \mathrm{C} ;{ }^{1} \mathrm{H}-\mathrm{NMR}(400 \mathrm{MHz}, \mathrm{DMSO}) \delta 8.42(\mathrm{dd}, J=9.7,2.1 \mathrm{~Hz}, 1 \mathrm{H}), 8.31(\mathrm{~d}, J=8.7 \mathrm{~Hz}, 1 \mathrm{H})$, 7.95 (ddd, $J=26.6,16.0,7.9 \mathrm{~Hz}, 4 \mathrm{H}) .{ }^{13} \mathrm{C}-\mathrm{NMR}(101 \mathrm{MHz}, \mathrm{DMSO}) \delta 164.72$ (s), 163.70 (s), 158.45 (s), 155.91 (s), 148.65 (s), 137.06 (s), 134.29 (s), 131.84 (s), 130.71 (s), 127.86 (s), 125.75 (s), 123.36 (s), 120.71 (s), 113.21 (s). HR-MS (ESI) $[\mathrm{M}+\mathrm{H}]^{+}$calcd for $\mathrm{C}_{14} \mathrm{H}_{6} \mathrm{ClFN}_{2} \mathrm{O}_{4}$ : 321.0000 , found: 321.0059 .

Data for 31 (4-chloro-2-(4-phenoxyphenyl)isoindoline-1,3-dione): white solid; yield, 86\%; m.p.: $172-173{ }^{\circ} \mathrm{C} ;{ }^{1} \mathrm{H}-\mathrm{NMR}\left(400 \mathrm{MHz}, \mathrm{CDCl}_{3}\right) \delta 8.05-7.98(\mathrm{~m}, 1 \mathrm{H}), 7.86(\mathrm{~d}, J=4.3 \mathrm{~Hz}, 2 \mathrm{H}), 7.52(\mathrm{dd}, J=4.7$, 
$4.0 \mathrm{~Hz}, 4 \mathrm{H}), 7.35-7.14(\mathrm{~m}, 5 \mathrm{H}) \cdot{ }^{13} \mathrm{C}-\mathrm{NMR}\left(101 \mathrm{MHz}, \mathrm{CDCl}_{3}\right) \delta 165.97$ (s), 164.98 (s), 157.39 (s), 156.44 (s), $136.16(\mathrm{~s}), 135.29$ (s), 133.86 (s), 131.92 (s), 129.94 (s), 128.07 (s), 127.40 (s), 125.97 (s), 123.97 (s), 122.25 (s), 119.60 (s), 118.81 (s). HR-MS (ESI) [M + H] calcd for $\mathrm{C}_{20} \mathrm{H}_{12} \mathrm{ClNO}_{3}: 350.0506$, found: 350.0580 .

Data for 3m (3-(4-chloro-1,3-dioxoisoindolin-2-yl)benzamide): light yellow solid; yield, 84\%; m.p.: 252-253 ${ }^{\circ} \mathrm{C} ;{ }^{1} \mathrm{H}-\mathrm{NMR}(400 \mathrm{MHz}, \mathrm{DMSO}) \delta 8.09(\mathrm{~s}, 1 \mathrm{H}), 8.03-7.83(\mathrm{~m}, 5 \mathrm{H}), 7.62(\mathrm{~s}, 2 \mathrm{H}), 7.53(\mathrm{~s}, 1 \mathrm{H})$. ${ }^{13} \mathrm{C}-\mathrm{NMR}\left(101 \mathrm{MHz}, \mathrm{CDCl}_{3}\right) \delta 172.19(\mathrm{~s}), 170.82(\mathrm{~s}), 169.85(\mathrm{~s}), 141.41$ (s), $141.12(\mathrm{~s}), 140.34(\mathrm{~s}), 139.16(\mathrm{~s})$, 136.99 (s), 135.44 (s), 135.05 (s), 134.05 (s), 132.53 (s), 132.23 (s), 132.10 (s), 127.60 (s). HR-MS (ESI) $[\mathrm{M}+\mathrm{H}]^{+}$calcd for $\mathrm{C}_{15} \mathrm{H}_{9} \mathrm{ClN}_{2} \mathrm{O}_{3}: 301.0302$, found: 301.0380 .

Data for 3n (2-(4-(tert-butyl)phenyl)-4-chloroisoindoline-1,3-dione): white solid; yield, 52\%; m.p.: 178-179 ${ }^{\circ} \mathrm{C} ;{ }^{1} \mathrm{H}-\mathrm{NMR}\left(400 \mathrm{MHz}, \mathrm{CDCl}_{3}\right) \delta 7.93-7.82(\mathrm{~m}, 1 \mathrm{H}), 7.70(\mathrm{~d}, J=3.8 \mathrm{~Hz}, 2 \mathrm{H}), 7.52(\mathrm{~d}, J=8.5 \mathrm{~Hz}$, 2H), $7.35(\mathrm{~d}, J=8.4 \mathrm{~Hz}, 2 \mathrm{H}), 1.36(\mathrm{~s}, 9 \mathrm{H}) .{ }^{13} \mathrm{C}-\mathrm{NMR}\left(101 \mathrm{MHz}, \mathrm{CDCl}_{3}\right) \delta 166.02(\mathrm{~s}), 165.01(\mathrm{~s}), 151.35(\mathrm{~s})$, 136.08 (s), 135.21 (s), 133.94 (s), 131.84 (s), 128.59 (s), 127.47 (s), 126.18 (s), 126.04 (s), 122.21 (s), 34.76 (s), 31.32 (s). HR-MS (ESI) [M + H] ${ }^{+}$calcd for $\mathrm{C}_{18} \mathrm{H}_{16} \mathrm{ClNO}_{2}$ : 314.0870, found: 314.0947.

Data for 3o (4-chloro-2-(3-fluoro-2-methoxyphenyl)isoindoline-1,3-dione): white solid; yield, 87\%; m.p.: $153-154{ }^{\circ} \mathrm{C}^{1}{ }^{1} \mathrm{H}-\mathrm{NMR}\left(400 \mathrm{MHz}, \mathrm{CDCl}_{3}\right) \delta$ 8.10-7.99 (m, 1H), 7.97-7.81 (m, 2H), 7.46-7.37 (m, 1H), $7.30(\mathrm{td}, J=8.1,5.2 \mathrm{~Hz}, 1 \mathrm{H}), 7.26-7.15(\mathrm{~m}, 1 \mathrm{H}), 4.14(\mathrm{~d}, J=2.4 \mathrm{~Hz}, 3 \mathrm{H}) \cdot{ }^{13} \mathrm{C}-\mathrm{NMR}\left(101 \mathrm{MHz}, \mathrm{CDCl}_{3}\right) \delta$ $165.63(\mathrm{~s}), 164.59$ (s), 156.89 (s), $154.43(\mathrm{~s}), 144.39$ (s), 136.11 (s), 135.25 (s), $134.10(\mathrm{~s}), 131.93$ (s), 127.76 (s), 125.01 (s), 123.03 (s), 122.32 (s), 118.40 (s), 61.42 (s). HR-MS (ESI) [M + H] $]^{+}$calcd for $\mathrm{C}_{15} \mathrm{H}_{9} \mathrm{ClFNO}_{3}$ : 306.0255, found: 306.0331 .

Data for 3p (4-chloro-2-(5-methylthiazol-2-yl)isoindoline-1,3-dione): yellow solid; yield, 30\%; m.p.: $143-144{ }^{\circ} \mathrm{C} ;{ }^{1} \mathrm{H}-\mathrm{NMR}\left(400 \mathrm{MHz}, \mathrm{CDCl}_{3}\right) \delta 7.83(\mathrm{dd}, J=63.0,3.8 \mathrm{~Hz}, 3 \mathrm{H}), 6.93(\mathrm{~s}, 1 \mathrm{H}), 2.52(\mathrm{~s}$, 3H). ${ }^{13} \mathrm{C}-\mathrm{NMR}\left(101 \mathrm{MHz}, \mathrm{CDCl}_{3}\right) \delta 163.32(\mathrm{~s}), 162.62$ (s), 150.64 (s), 150.45 (s), 136.77 (s), $135.92(\mathrm{~s})$, $133.30(\mathrm{~s}), 132.62(\mathrm{~s}), 126.87$ (s), 122.81 (s), 113.01 (s), 17.48 (s). HR-MS (ESI) [M + H] ${ }^{+}$calcd for $\mathrm{C}_{12} \mathrm{H}_{7} \mathrm{ClN}_{2} \mathrm{O}_{2} \mathrm{~S}: 278.9917$, found: 278.9988 .

Data for 3q (methyl 5-fluoro-2-(4-fluoro-1,3-dioxoisoindolin-2-yl)benzoate): white solid; yield, 57\%; m.p.: $179-180{ }^{\circ} \mathrm{C} ;{ }^{1} \mathrm{H}-\mathrm{NMR}\left(400 \mathrm{MHz}, \mathrm{CDCl}_{3}\right) \delta 8.09-7.65(\mathrm{~m}, 3 \mathrm{H}), 7.56-7.29(\mathrm{~m}, 3 \mathrm{H}), 3.79(\mathrm{~d}$, $J=3.0 \mathrm{~Hz}, 3 \mathrm{H}) .{ }^{13} \mathrm{C}-\mathrm{NMR}\left(101 \mathrm{MHz}, \mathrm{CDCl}_{3}\right) \delta 166.33(\mathrm{~s}), 163.96(\mathrm{~s}), 159.24(\mathrm{~s}), 156.59(\mathrm{~s}), 136.94(\mathrm{~s})$, 134.23 (s), 132.38 (s), 129.82 (s), 127.45 (s), 122.87 (s), 122.68 (s), 120.38 (s), 120.10 (s), 119.00 (s), 118.75 (s), 52.72 (s). HR-MS (ESI) [M + H] $]^{+}$calcd for $\mathrm{C}_{16} \mathrm{H}_{9} \mathrm{~F}_{2} \mathrm{NO}_{4}$ : 318.0500, found: 318.0574 .

Data for 3r (2-(4-bromo-2,6-difluorophenyl)-4-fluoroisoindoline-1,3-dione): shite solid; yield, 78\%; m.p.: $140-142{ }^{\circ} \mathrm{C} ;{ }^{1} \mathrm{H}-\mathrm{NMR}\left(400 \mathrm{MHz}, \mathrm{CDCl}_{3}\right) \delta 7.90-7.79(\mathrm{~m}, 2 \mathrm{H}), 7.52(\mathrm{t}, J=8.0 \mathrm{~Hz}, 1 \mathrm{H}), 7.32(\mathrm{~d}$, $J=6.9 \mathrm{~Hz}, 2 \mathrm{H}) .{ }^{13} \mathrm{C}-\mathrm{NMR}\left(101 \mathrm{MHz}, \mathrm{CDCl}_{3}\right) \delta 164.25$ (s), $161.90(\mathrm{~s}), 160.05$ (s), 159.36 (s), 156.70 (s), 137.41 (s), 133.92 (s), 123.60 (s), 123.20 (s), 123.01 (s), 120.43 (s), 117.81 (s), 116.30 (s), 108.15 (s). HR-MS (ESI) $[\mathrm{M}+\mathrm{H}]^{+}$calcd for $\mathrm{C}_{14} \mathrm{H}_{5} \mathrm{BrF}_{3} \mathrm{NO}_{2}$ : 355.9456, found: 355.9522 .

Data for 3s (4-fluoro-2-(4-isopropylphenyl)isoindoline-1,3-dione): shite solid; yield, 79\%; m.p.: $116-117^{\circ} \mathrm{C} ;{ }^{1} \mathrm{H}-\mathrm{NMR}\left(400 \mathrm{MHz}, \mathrm{CDCl}_{3}\right) \delta 7.68(\mathrm{~d}, J=2.4 \mathrm{~Hz}, 2 \mathrm{H}), 7.34(\mathrm{ddd}, J=9.0,6.1,3.1 \mathrm{~Hz}, 1 \mathrm{H})$, $7.25(\mathrm{q}, J=8.6 \mathrm{~Hz}, 4 \mathrm{H}), 2.87(\mathrm{dt}, J=13.8,6.9 \mathrm{~Hz}, 1 \mathrm{H}), 1.19(\mathrm{~d}, J=6.9 \mathrm{~Hz}, 6 \mathrm{H}) .{ }^{13} \mathrm{C}-\mathrm{NMR}(101 \mathrm{MHz}$, $\left.\mathrm{CDCl}_{3}\right) \delta 166.33(\mathrm{~s}), 164.08(\mathrm{~s}), 159.21(\mathrm{~s}), 156.56(\mathrm{~s}), 149.16(\mathrm{~s}), 136.84(\mathrm{~s}), 134.03(\mathrm{~s}), 128.77(\mathrm{~s}), 127.28(\mathrm{~s})$, 126.44 (s), 122.59 (s), 119.94 (s), 33.94 (s), 23.91 (s). HR-MS (ESI) $[\mathrm{M}+\mathrm{H}]^{+}$calcd for $\mathrm{C}_{17} \mathrm{H}_{14} \mathrm{FNO}_{2}$ : 284.1009, found: 284.1086 .

Data for 3t (5-chloro-2-(3-fluoro-4-methylphenyl)isoindoline-1,3-dione): shite solid; yield, 79\%; m.p.: $178-179{ }^{\circ} \mathrm{C} ;{ }^{1} \mathrm{H}-\mathrm{NMR}\left(400 \mathrm{MHz}, \mathrm{CDCl}_{3}\right) \delta 7.96-7.85(\mathrm{~m}, 2 \mathrm{H}), 7.76(\mathrm{dd}, J=8.0,1.4 \mathrm{~Hz}, 1 \mathrm{H}), 7.31(\mathrm{t}$, $J=8.1 \mathrm{~Hz}, 1 \mathrm{H}), 7.14(\mathrm{~d}, J=8.8 \mathrm{~Hz}, 2 \mathrm{H}), 2.33(\mathrm{~s}, 3 \mathrm{H}) .{ }^{13} \mathrm{C}-\mathrm{NMR}\left(101 \mathrm{MHz}, \mathrm{CDCl}_{3}\right) \delta 166.07(\mathrm{~s}), 165.76(\mathrm{~s})$, $162.14(\mathrm{~s}), 141.29$ (s), 134.61 (s), 133.29 (s), 131.68 (s), 129.67 (s), 125.10 (s), $124.26(\mathrm{~s}), 121.79$ (s), 113.65 (s), 113.40 (s), 99.99 (s), 14.41 (s). HR-MS (ESI) [M + H] ${ }^{+}$calcd for $\mathrm{C}_{15} \mathrm{H}_{9} \mathrm{ClFNO}_{2}: 290.0306$, found: 290.0376.

Data for 3u (5-chloro-2-(4-isopropylphenyl)isoindoline-1,3-dione): white solid; yield, 70\%; m.p.: 137-138 ${ }^{\circ} \mathrm{C} ;{ }^{1} \mathrm{H}-\mathrm{NMR}\left(400 \mathrm{MHz}, \mathrm{CDCl}_{3}\right) \delta 7.90(\mathrm{dd}, J=13.7,4.7 \mathrm{~Hz}, 2 \mathrm{H}), 7.74(\mathrm{dd}, J=8.0,1.8 \mathrm{~Hz}, 1 \mathrm{H})$, $7.34(\mathrm{~d}, J=8.6 \mathrm{~Hz}, 4 \mathrm{H}), 2.97(\mathrm{dt}, J=13.8,6.9 \mathrm{~Hz}, 1 \mathrm{H}), 1.28(\mathrm{~d}, J=6.9 \mathrm{~Hz}, 6 \mathrm{H}) .{ }^{13} \mathrm{C}-\mathrm{NMR}(101 \mathrm{MHz}$, $\left.\mathrm{CDCl}_{3}\right) \delta 166.50(\mathrm{~s}), 166.19(\mathrm{~s}), 149.16(\mathrm{~s}), 141.06(\mathrm{~s}), 134.41(\mathrm{~s}), 133.50(\mathrm{~s}), 129.88(\mathrm{~s}), 128.91(\mathrm{~s}), 127.29(\mathrm{~s})$, 
126.34 (s), 124.97 (s), 124.14 (s), 33.94 (s), 23.91 (s). HR-MS (ESI) $[\mathrm{M}+\mathrm{H}]^{+}$calcd for $\mathrm{C}_{17} \mathrm{H}_{14} \mathrm{ClNO}_{2}$ : 300.0713, found: 300.0791 .

Data for 3v (5-chloro-2-(1-methyl-1H-pyrazol-5-yl)isoindoline-1,3-dione): white solid; yield, 76\%; m.p.: $175-176{ }^{\circ} \mathrm{C} ;{ }^{1} \mathrm{H}-\mathrm{NMR}\left(400 \mathrm{MHz}, \mathrm{CDCl}_{3}\right) \delta 7.95(\mathrm{dd}, J=12.1,4.7 \mathrm{~Hz}, 2 \mathrm{H}), 7.83(\mathrm{dd}, J=8.0,1.6$ $\mathrm{Hz}, 1 \mathrm{H}), 7.61(\mathrm{~d}, J=1.9 \mathrm{~Hz}, 1 \mathrm{H}), 6.33(\mathrm{~d}, J=1.9 \mathrm{~Hz}, 1 \mathrm{H}), 3.78(\mathrm{~s}, 3 \mathrm{H}) \cdot{ }^{13} \mathrm{C}-\mathrm{NMR}\left(101 \mathrm{MHz}, \mathrm{CDCl}_{3}\right) \delta$ 165.09 (s), 164.81 (s), 141.88 (s), 138.85 (s), 135.10 (s), 133.20 (s), 129.59 (s), 129.20 (s), 125.55 (s), 124.69 (s), 104.63 (s), 36.47 (s). HR-MS (ESI) [M + H] $]^{+}$calcd for $\mathrm{C}_{12} \mathrm{H}_{8} \mathrm{ClN}_{3} \mathrm{O}_{2}: 262.0305$, found: 262.0384 .

Data for 3w (5-chloro-2-(thiophen-2-ylmethyl)isoindoline-1,3-dione): white solid; yield, 79\%; m.p.: 93-94 ${ }^{\circ} \mathrm{C} ;{ }^{1} \mathrm{H}-\mathrm{NMR}\left(400 \mathrm{MHz}, \mathrm{CDCl}_{3}\right) \delta 7.89(\mathrm{dd}, J=11.9,4.9 \mathrm{~Hz}, 2 \mathrm{H}), 7.78(\mathrm{~d}, J=8.0 \mathrm{~Hz}, 1 \mathrm{H}), 7.39-7.22$ $(\mathrm{m}, 2 \mathrm{H}), 7.04(\mathrm{dd}, J=6.0,2.4 \mathrm{~Hz}, 1 \mathrm{H}), 5.11(\mathrm{~s}, 2 \mathrm{H}) .{ }^{13} \mathrm{C}-\mathrm{NMR}\left(101 \mathrm{MHz}, \mathrm{CDCl}_{3}\right) \delta 166.60(\mathrm{~s}), 166.27(\mathrm{~s})$, 140.81 (s), 137.67 (s), 134.12 (s), 133.72 (s), 130.11 (s), 127.90 (s), 126.94 (s), 126.05 (s), 124.70 (s), 123.93 (s), 35.91 (s). HR-MS (ESI) [M + H] $]^{+}$calcd for $\mathrm{C}_{13} \mathrm{H}_{8} \mathrm{ClNO}_{2} \mathrm{~S}: 277.9964$, found: 278.0032 .

Data for 3x (2-(4-isopropylphenyl)-5-methylisoindoline-1,3-dione): white solid; yield, 47\%; m.p.: 161-162 ${ }^{\circ} \mathrm{C} ;{ }^{1} \mathrm{H}-\mathrm{NMR}\left(400 \mathrm{MHz}, \mathrm{CDCl}_{3}\right) \delta 7.82(\mathrm{~d}, J=7.6 \mathrm{~Hz}, 1 \mathrm{H}), 7.75(\mathrm{~s}, 1 \mathrm{H}), 7.57(\mathrm{~d}, J=7.5 \mathrm{~Hz}$, $1 \mathrm{H}), 7.35(\mathrm{~s}, 4 \mathrm{H}), 2.96(\mathrm{dt}, J=13.7,6.9 \mathrm{~Hz}, 1 \mathrm{H}), 2.54(\mathrm{~s}, 3 \mathrm{H}), 1.28(\mathrm{~d}, J=6.9 \mathrm{~Hz}, 6 \mathrm{H}) .{ }^{13} \mathrm{C}-\mathrm{NMR}(101$ $\left.\mathrm{MHz}, \mathrm{CDCl}_{3}\right) \delta 167.66(\mathrm{~s}), 167.54(\mathrm{~s}), 148.77$ (s), 145.67 (s), 134.89 (s), 132.22 (s), 129.32 (s), 129.25 (s), 127.19 (s), 126.44 (s), 124.21 (s), 123.62 (s), 33.94 (s), 23.94 (s), 22.07 (s). HR-MS (ESI) [M + H] ${ }^{+}$calcd for $\mathrm{C}_{18} \mathrm{H}_{17} \mathrm{NO}_{2}$ : 280.1259, found: 280.1338 .

Data for 3y (2-(4-isopropylphenyl)-5-nitroisoindoline-1,3-dione): white solid; yield, 57\%; m.p.: 179-180 ${ }^{\circ} \mathrm{C} ;{ }^{1} \mathrm{H}-\mathrm{NMR}\left(400 \mathrm{MHz}, \mathrm{CDCl}_{3}\right) \delta 8.77(\mathrm{~d}, J=1.7 \mathrm{~Hz}, 1 \mathrm{H}), 8.67(\mathrm{dd}, J=8.1,1.9 \mathrm{~Hz}, 1 \mathrm{H}), 8.15(\mathrm{~d}$, $J=8.1 \mathrm{~Hz}, 1 \mathrm{H}), 7.44-7.30(\mathrm{~m}, 4 \mathrm{H}), 3.09-2.84(\mathrm{~m}, 1 \mathrm{H}), 1.29(\mathrm{~d}, J=6.9 \mathrm{~Hz}, 6 \mathrm{H}) \cdot{ }^{13} \mathrm{C}-\mathrm{NMR}(101 \mathrm{MHz}$, $\left.\mathrm{CDCl}_{3}\right) \delta 165.11(\mathrm{~s}), 151.97(\mathrm{~s}), 149.66(\mathrm{~s}), 136.21(\mathrm{~s}), 133.21(\mathrm{~s}), 129.56(\mathrm{~s}), 128.51(\mathrm{~s}), 127.44(\mathrm{~s}), 126.23(\mathrm{~s})$, 124.97 (s), 119.14 (s), 33.97 (s), 23.90 (s). HR-MS (ESI) $[\mathrm{M}+\mathrm{H}]^{+}$calcd for $\mathrm{C}_{17} \mathrm{H}_{14} \mathrm{~N}_{2} \mathrm{O}_{4}$ : 311.0954 , found: 311.1032 .

\subsection{Herbicidal Activity}

The herbicidal activities of the test compounds against B. campestris, A. retroflexus and D. sanguinalis were evaluated by the small cup method and foliar spray method at $200 \mathrm{mg} / \mathrm{L}$, according the following procedure [33,34]. With respect to the small cup method, generally, the test compound (20 $\mathrm{mg}$ ) was dissolved in $N, N$-dimethylformamide (DMF) $(1 \mathrm{~mL})$ and then diluted in water containing $0.1 \%$ Tween 80 to a final concentration of $200 \mathrm{mg} / \mathrm{L}$. The controls were treated with the same solution but without any test compound. A piece of filter paper in a $50 \mathrm{~mL}$ beaker was treated with the test compound solution $(1 \mathrm{~mL})$, and then 10 seeds that were soaked in water for $24 \mathrm{~h}$ were added. All treatments were repeated three times.

The pre- and post-emergence herbicidal activities of the title compounds were evaluated at an application rate of $90 \mathrm{~g}$ ai/ha in a greenhouse according to a reported method $[35,36]$. Nine seeds of these plants (A. retroflexus seeds, B. campestris seeds or D. sanguinalis seeds) were sown at a depth of $5 \mathrm{~mm}$ in a mixture of vermiculite/nutrient-enriched soil $(1: 1, \mathrm{~m} / \mathrm{m})$ with some water at $4 \mathrm{~cm}$ below the surface and then cultivated at $20-25^{\circ} \mathrm{C}$. To test the pre-emergence herbicidal activities, the abovementioned soil was sprayed with the title compound solution before germination, and the results were determined after two weeks. To test the post-emergence herbicidal activities, plants at the 2-4 leaf stage after germination were treated with the test compound solution by a walking spray tower and then cultivated for one week. The percentage of herbicidal activity was calculated by comparing the fresh weight of the growth-inhibited plants with that of the healthy control plants, where completely inhibited growth was set as 100 and the healthy control was set as 0 .

\subsection{PPO Enzyme Assays}

To further explore the mode of action of these target compounds, 3a was selected as a representative to confirm whether it can act on PPO. Briefly, the procedures were followed as described here. D. sanguinalis plants were treated with $90 \mathrm{~g}$ ai/ha of compound $\mathbf{3 a}$ and Flumioxazin by 
the post-emergence method. After 3 days, $D$. sanguinalis leaves $(0.2 \mathrm{~g})$ were collected and dissolved in extraction medium $(1.5 \mathrm{~mL})$ in an ice bath, and the mixture was then centrifuged at $4{ }^{\circ} \mathrm{C} \times 12,000 \mathrm{rpm}$ for $15 \mathrm{~min}$. A polyphenol oxidase kit (G0113W, Suzhou Grace Bio-technology Co., Ltd., Suzhou, China) was obtained from commercial sources to determine the PPO activity. The change in absorbance (every $5 \mathrm{~min}$ ) was measured on a POLARstar Optima/Galaxy instrument (BMG) (Shanghai Microplate Co., Ltd., Shanghai, China) at $420 \mathrm{~nm}$ [37-39]. One unit of PPO activity was defined as a change in absorbance of 0.01 per minute.

\section{Conclusions}

In summary, a series of phthalimide derivatives were designed by molecular docking and by using Flumioxazin as a lead, then synthesized and characterized by NMR, HR-MS, and the typical crystal structure was determined by $\mathrm{X}$-ray diffraction. The herbicidal activities of these compounds were assessed against $B$. campestris, $A$. retroflexus and $D$. sanguinalis, by the small cup, pre-emergence, and post-emergence methods, respectively. Most of the synthesized compounds exhibited good to excellent herbicidal activities, and especially 3 a displayed the same efficacy against $A$. retroflexus and B. campestris to commercial standards of Flumioxazin. Further PPO activity assays confirmed that the mode of action of $3 a$ is similar to PPO inhibitors. These results suggest that our molecular design strategy is effective.

Author Contributions: The experiments were conceived and planned by W.G., L.C. and J.Z., the manuscript was written by W.G., L.C. and J.H. Experiments were performed by W.G., X.L., D.R., S.S. and Y.W. The final version of this manuscript has been read and approved by all authors.

Funding: This research was in financial supported by the National Natural Science Foundation of China (Nos. 31871981); the starting Scientific Research Foundation for the introduced talents of Hebei Agricultural University (Nos. 201842); Youth Natural Science Foundation of Hebei Province (Nos. B2019204030); Modern Agriculture Industry Technology System Innovation Team of Phase II of Hebei Province (Nos. HB2018020205).

Conflicts of Interest: Authors hereby declare that there are no financial/commercial conflict of interest.

\section{References}

1. Poulson, R.; Polglase, W. The enzymic conversion of protoporphyrinogen IX to protoporphyrin IX. Protoporphyrinogen oxidase activity in mitochondrial extracts of Saccharomyces cerevisiae. J. Biol. Chem. 1975, 250, 1269-1274.

2. Poulson, R. The enzymic conversion of protoporphyrinogen IX to protoporphyrin IX in mammalian mitochondria. J. Biol. Chem. 1976, 251, 3730-3733.

3. Ajioka, R.S.; Phillips, J.D.; Kushner, J.P. Biosynthesis of heme in mammals. Biochim. Biophys. Acta 2006, 1763, 723-736. [CrossRef]

4. Heinemann, I.U.; Jahn, M.; Jahn, D. The biochemistry of heme biosynthesis. Biochem. Biophys. 2008, 474, 238-251. [CrossRef]

5. Kato, K.; Tanaka, R.; Sano, S.; Tanaka, A.; Hosaka, H. Identification of a gene essential for protoporphyrinogen IX oxidase activity in the cyanobacterium Synechocystis sp. PCC6803. Natl. Acad. Sci. 2010, 107, 16649-16654. [CrossRef]

6. Wang, B.; Wen, X.; Qin, X.; Wang, Z.; Tan, Y.; Shen, Y.; Xi, Z. Quantitative structural insight into human variegate porphyria disease. J. Biol. Chem. 2013, 288, 11731-11740. [CrossRef]

7. Wang, B.; Wen, X.; Xi, Z. Molecular simulations bring new insights into protoporphyrinogen IX oxidase/protoporphyrinogen IX interaction modes. Mol. Inform. 2016, 35, 476-482. [CrossRef]

8. Dayan, F.E.; Barker, A.; Tranel, P.J. Origins and structure of chloroplastic and mitochondrial plant protoporphyrinogen oxidases: Implications for the evolution of herbicide resistance. Pest Manage. Sci. 2018, 74, 2226-2234. [CrossRef] [PubMed]

9. Qin, X.; Sun, L.; Wen, X.; Yang, X.; Tan, Y.; Jin, H.; Cao, Q.; Zhou, W.; Xi, Z.; Shen, Y. Structural insight into unique properties of protoporphyrinogen oxidase from Bacillus subtilis. J. Struct. Biol. 2010, 170, 76-82. [CrossRef] [PubMed] 
10. Sherman, T.D.; Becerril, J.M.; Matsumoto, H.; Duke, M.V.; Jacobs, J.M.; Jacobs, N.J.; Duke, S.O. Physiological basis for differential sensitivities of plant species to protoporphyrinogen oxidase-inhibiting herbicides. Plant Physiol. 1991, 97, 280-287. [CrossRef] [PubMed]

11. Hao, G.F.; Zuo, Y.; Yang, S.G.; Yang, G.F. Protoporphyrinogen oxidase inhibitor: An ideal target for herbicide discovery. CHIMIA Int. J. Chem. 2011, 65, 961-969. [CrossRef] [PubMed]

12. Meazza, G.; Bettarini, F.; Porta, P.L.; Piccardi, P.; Signorini, E.; Portoso, D.; Fornara, L. Synthesis and herbicidal activity of novel heterocyclic protoporphyrinogen oxidase inhibitors. Pest Manage. Sci. 2004, 60, 1178-1188. [CrossRef] [PubMed]

13. Jiang, L.L.; Tan, Y.; Zhu, X.L.; Wang, Z.F.; Zuo, Y.; Chen, Q.; Xi, Z.; Yang, G.F. Design, synthesis, and 3D-QSAR analysis of novel 1, 3, 4-oxadiazol-2 (3H)-ones as protoporphyrinogen oxidase inhibitors. J. Agric. Food Chem. 2009, 58, 2643-2651. [CrossRef] [PubMed]

14. Jiang, L.L.; Zuo, Y.; Wang, Z.F.; Tan, Y.; Wu, Q.Y.; Xi, Z.; Yang, G.F. Design and syntheses of novel N-(benzothiazol-5-yl)-4,5,6,7-tetrahydro-1H-isoindole-1,3(2H)-dione and N-(benzothiazol-5-yl) isoindoline-1,3-dione as potent protoporphyrinogen oxidase inhibitors. J. Agric. Food Chem. 2011, 59, 6172-6179. [CrossRef] [PubMed]

15. Zuo, Y.; Wu, Q.; Su, S.W.; Niu, C.W.; Xi, Z.; Yang, G.F. Synthesis, herbicidal activity, and QSAR of novel N-benzothiazolyl-pyrimidine-2,4-diones as protoporphyrinogen oxidase inhibitors. J. Agric. Food Chem. 2016, 64, 552-562. [CrossRef] [PubMed]

16. Kwon, J.W.; Armbrust, K.L.; Grey, T.L. ydrolysis and photolysis of flumioxazin in aqueous buffer solutions. Pest Manage. Sci. 2004, 60, 939-943. [CrossRef]

17. Arakawa, A.; Otani, M.; Iwashita, K.; Yamazaki, K. Molecular dynamics mechanism to generate species differences in inhibition of protoporphyrinogen oxidase by flumioxazin. Comp. Toxi. 2017, 1, 12-21. [CrossRef]

18. Dayan, F.E.; Armstrong, B.M.; Weete, J.D. Inhibitory activity of sulfentrazone and its metabolic derivatives on soybean (Glycine max) protoporphyrinogen oxidase. J. Agric. Food Chem. 1998, 46, 2024-2029. [CrossRef]

19. Grossmann, K.; Niggeweg, R.; Christiansen, N.; Looser, R.; Ehrhardt, T. The herbicide saflufenacil (Kixor ${ }^{\mathrm{TM}}$ ) is a new inhibitor of protoporphyrinogen IX oxidase activity. Weed Sci. 2010, 58, 1-9. [CrossRef]

20. Chen, L.; Zhang, Y.; Yu, H.; Cui, D.; Li, B. Tetrahydrophthalimidobenzoates as protoporphyrinogen IX oxidase inhibiting herbicides. Pestic. Biochem. Physiol. 2017, 139, 40-45. [CrossRef]

21. Chaudhari, R.; Tan, Z.; Huang, B.; Zhang, S. Computational polypharmacology: A new paradigm for drug discovery. Expert Opin. Drug Discov. 2017, 12, 279-291. [CrossRef] [PubMed]

22. Huang, M.Z.; Luo, F.X.; Mo, H.B.; Ren, Y.G.; Wang, X.G.; Ou, X.M.; Lei, M.X.; Liu, A.P.; Huang, L.; Xu, M.C. Synthesis and herbicidal activity of isoindoline-1,3-dione substituted benzoxazinone derivatives containing a carboxylic ester group. J. Agric. Food Chem. 2009, 57, 9585-9592. [CrossRef] [PubMed]

23. Jeschke, P. The Unique Role of Halogen Substituents in the Design of Modern Crop Protection Compounds; Wiley Online Library: Weinheim, Germany, 2012.

24. Trott, O.; Olson, A.J. AutoDock Vina: Improving the speed and accuracy of docking with a new scoring function, efficient optimization, and multithreading. J. Comput. Chem. 2010, 31, 455-461. [CrossRef] [PubMed]

25. Tanchuk, V.Y.; Tanin, V.O.; Vovk, I.; Poda, G. A new improved hybrid scoring function for molecular docking and scoring based on autodock and autodock vina. Chem. Biol. Drug Des. 2016, 87, 618-625. [CrossRef] [PubMed]

26. Chaudhari, R.; Li, Z. PyMine: A PyMOL plugin to integrate and visualize data for drug discovery. BMC Res. Notes 2015, 8, 517. [CrossRef] [PubMed]

27. Giovani, S.; Penzo, M.; Butini, S.; Brindisi, M.; Gemma, S.; Novellino, E.; Campiani, G.; Blackman, M.J.; Brogi, S. Plasmodium falciparum subtilisin-like protease 1: Discovery of potent difluorostatone-based inhibitors. RSC Adv. 2015, 5, 22431-22448. [CrossRef]

28. Garcia-Barrantes, P.M.; Cho, H.P.; Metts, A.M.; Blobaum, A.L.; Niswender, C.M.; Conn, P.J.; Lindsley, C.W. Lead optimization of the VU0486321 series of mGlu1 PAMs. Part 2: SAR of alternative 3-methyl heterocycles and progress towards an in vivo tool. Bioorg. Med. Chem. Lett. 2016, 26, 751-756. [CrossRef]

29. Cheng, H.; Fu, Y.; Chang, Q.; Zhang, N.; Bu, M.W.; Niu, Y.; Wu, Q.Y.; Chen, C.; Verpoort, F. Synthesis, biochemical evaluation and computational simulations of new cytochrome bc1 complex inhibitors based on N-(4-aryloxyphenyl) phthalimides. Chin. Chem. Lett. 2018, 29, 1897-1900. [CrossRef] 
30. Yoon, H.S.; Hong, W.P.; Kang, Y.J.; Kim, J.J.; Han, S.H. Preparation of Indolocarbazole Compound for Organic Light Emitting Device. KR 2019101894, 2 September 2019.

31. Barchín, B.M.; Cuadro, A.M.; Alvarez-Builla, J. Microwave-assisted parallel synthesis of a 2-aryl-1Hisoindole-1,3-dione library. Synlett 2002, 2, 343-345. [CrossRef]

32. Fujimaki, N.; Nakagomi, M.; Shudo, K. Preparation of Aromatic Amide Derivatives Affecting Gene Transcription Process. WO 2009107762, 3 September 2009.

33. Wang, D.W.; Lin, H.Y.; Cao, R.J.; Chen, T.; Wu, F.X.; Hao, G.F.; Chen, Q.; Yang, W.C.; Yang, G.F. Synthesis and herbicidal activity of triketone-quinoline hybrids as novel 4-hydroxyphenylpyruvate dioxygenase inhibitors. J. Agric. Food Chem. 2015, 63, 5587-5596. [CrossRef]

34. Huo, J.Q.; Zhao, B.; Zhang, Z.; Xing, J.H.; Zhang, J.L.; Dong, J.G.; Fan, Z.J. Structure-Based Discovery and Synthesis of Potential Transketolase Inhibitors. Molecules 2018, 23, 2116. [CrossRef] [PubMed]

35. Sartori, S.K.; Alvarenga, E.S.; Franco, C.A.; Ramos, D.S.; Oliveira, D.F. One-pot synthesis of anilides, herbicidal activity and molecular docking study. Pest Manage. Sci. 2018, 74, 1637-1645. [CrossRef] [PubMed]

36. Zhao, A.; Liu., S.; Zhu., Y.; Wang., T.; Luo., J. Designed, Synthesis and Herbicidal Activity of Novel 2-Alkylthio-thieno[2,3-d] pyrimidin-4-ones. Chin. J. Chem. 2017, 37, 1877. [CrossRef]

37. Aquino-Bolaños, E.N.; Mercado-Silva, E. Effects of polyphenol oxidase and peroxidase activity, phenolics and lignin content on the browning of cut jicama. Postharvest Biol. Technol. 2004, 33, 275-283. [CrossRef]

38. Queiroz, C.; da-Silva, A.J.R.; Lopes, M.L.M.; Fialho, E.; Valente-Mesquita, V.L. Polyphenol oxidase activity, phenolic acid composition and browning in cashew apple (Anacardium occidentale, L.) after processing. Food Chem. 2011, 125, 128-132. [CrossRef]

39. Ciou, J.Y.; Lin, H.H.; Chiang, P.Y.; Wang, C.C.; Charles, A.L. The role of polyphenol oxidase and peroxidase in the browning of water caltrop pericarp during heat treatment. Food Chem. 2011, 127, 523-527. [CrossRef]

(C) 2019 by the authors. Licensee MDPI, Basel, Switzerland. This article is an open access article distributed under the terms and conditions of the Creative Commons Attribution (CC BY) license (http://creativecommons.org/licenses/by/4.0/). 